\title{
Application of Aberration-Corrected Transmission Electron Microscopy to Materials Science
}

\author{
K. Urban and M. Lentzen \\ Institute for Solid State Research, Research Center Jülich GmbH, D-52425 Jülich, Germany
}

Recently an electromagnetic hexapole system for the correction of the spherical aberration of the objective lens of a $200 \mathrm{kV}$ transmission electron microscope was constructed by Haider and coworkers [1]. Integrating this system in a Philips CM200 FEG ST an increase in point resolution from 0.24 to better than $0.13 \mathrm{~nm}$ could be demonstrated [2]. However, transmission electron microscopy involving an aberration corrector offers additional features of interest for materials science application.

By appropriately exciting the hexapole elements it is possible to adjust specific values of the spherical aberration coefficient $C_{\mathrm{S}}$ ranging from the value of the original objective lens to zero. Thus $C_{\mathrm{S}}$ enters as an additional variable, besides the defocus value $Z$, into the wave aberration function

$\chi(\vec{g})=1 / 2 Z \lambda g^{2}+1 / 4 C_{S} \lambda^{3} g^{4}$,

where $\lambda$ is the electron wave length and $\vec{g}$ the spatial frequency.

Adjusting the corrector for $C_{\mathrm{S}}=0$ and applying $Z=0$ the phase-contrast term $-i \sin 2 \pi \chi(g)$ in the transfer function vanishes. On the other hand, the amplitude-contrast term $\cos 2 \pi \chi(g)$ adopts its maximum value 1 . This means that in the fully aberration-corrected mode the microscope images atomic structures by amplitude rather than by the usual phase-contrast [3]. In the electron exit-plane wave function local amplitude variations are produced by electron diffraction channeling. The projected atom columns are imaged by bright contrast on a dark background. As demonstrated by the simulation for Ge [110] in Fig. 1 maximum contrast occurs at thicknesses of half the extinction distance defined by the two most excited Bloch waves and odd multiples of it. This mode has the special advantage that contrast delocalization defined by the radius of the point-spread function ( $g_{\max }$ - information limit) [4]

$R=\max \left|\frac{\partial \chi}{\partial \vec{g}}\right|,\left[0, g_{\max }\right]$,

is zero and optimum imaging of defects, boundaries and interfaces can be achieved.

Besides this pure amplitude-contrast mode other imaging modes are available in which phase-contrast contributes to the image in addition. In order to determine the optimum values for $Z$ and $C_{\mathrm{s}}$ for this kind of imaging an optimization problem has to be solved which yields an optimum defocus and spherical-aberration setting. This pair of values represents the equivalent to Scherzer's defocus in id ealized weak-phase object imaging. The optimization problem arises from the fact that finite values for $Z$ and/or $C_{\mathrm{S}}$ induce contrast delocalization. A detailed treatment of this problem [3] yields for the optimum values yielding maximum phase contrast at a minimum of delocalization

$$
C_{S, o p t}=64 / 27 \lambda^{-3} g_{\max }^{-4} ; Z_{\text {opt }}=-16 / 9 \lambda g_{\max }^{-2}
$$

For the CM200 FEG ST one obtains for $\lambda=2.5 \mathrm{pm}$ and an information limit of $7.3 \mathrm{~nm}^{-1}$ the values $C_{\mathrm{S}, \mathrm{opt}}=53 \mu \mathrm{m}$ and $Z_{\mathrm{opt}}=-13.3 \mathrm{~nm}$. Under these conditions the contrast delocalization amounts to $R_{\mathrm{opt}}=0.081 \mathrm{~nm}$. In such a calculation, in the same way as in conventional transmission electron microscopy 
and the derivation of the idealized Scherzer formula, only phase shifts introduced by the electron optics are taken into account. Phase shifts already contained in the electron exit-plane wave function are not considered. In practice it has proven to be advantageous to take a through-focus series of images and to accompany the practical high-resolution work by image simulation or exit-wave function reconstruction. This not only allows to define the conditions for $\mathrm{Z}=0$, it also permits to select and understand the images taken under optimum conditions.

The following examples of images are taken at $Z=0 / C_{\mathrm{S}}=0$. Figure 2 (courtesy $\mathrm{K}$. Tillmann) shows an application for the determination of Al-concentration profiles in low-temperature GaAs/AlAs heterostructures. Due to the absence of contrast delocalization the profile can be directly derived from the (002) component of the Fourier transform of the image intensity distribution. Figure 3 (courtesy J.L. Jia) shows a tilt boundary in $(\mathrm{BaSr}) \mathrm{TiO}_{3}$ along the [100] zone axis demonstrating the high quality of delocalization-free interface imaging under amplitude contrast conditions. Figure 4 (courtesy J.L. Jia) demonstrates the high resolution of 0.14 $\mathrm{nm}$ in $(\mathrm{BaSr}) \mathrm{TiO}_{3}$ along the [110] zone axis.

\section{References}

[1] M. Haider, H. Rose, S. Uhlemann, E. Schwan, B. Kabius, K. Urban, Nature 392 (1998) 768.

[2] M. Haider, H. Rose, S. Uhlemann, E. Schwan, B. Kabius, K. Urban, Ultramicroscopy 75 (1998) 53.

[3] M. Lentzen, B. Jahnen, C.L. Jia, A. Thust, K. Tillmann, K. Urban, Ultramicroscopy, in press.

[4] H. Lichte, Ultramicroscopy 38 (1991) 13.

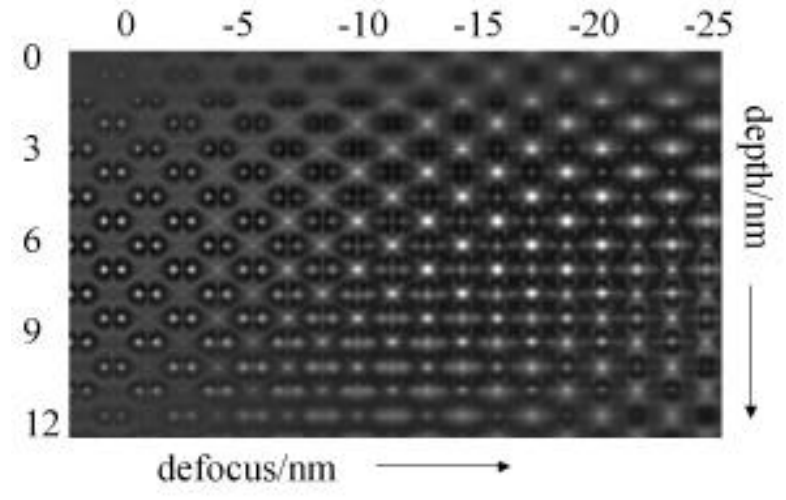

Fig.1 Calculated through-focus and through-thickness image series of Ge [110] for $C_{\mathrm{S}}=0$.



Fig. 3 Tilt boundary in $(\mathrm{BaSr}) \mathrm{TiO}_{3}$ at $Z=0$ and $C_{\mathrm{S}}=0$. Viewing direction [100].

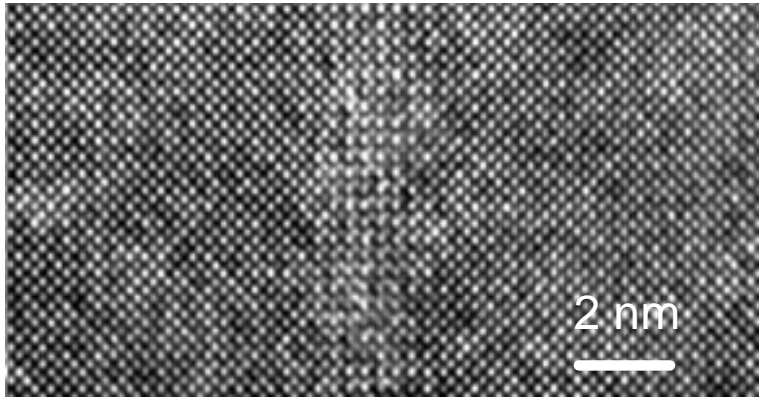

Fig. $2 \mathrm{GaAs} / \mathrm{AlAs}$ heterostructure at $\mathrm{Z}=0$ and $C_{\mathrm{S}}=0$.

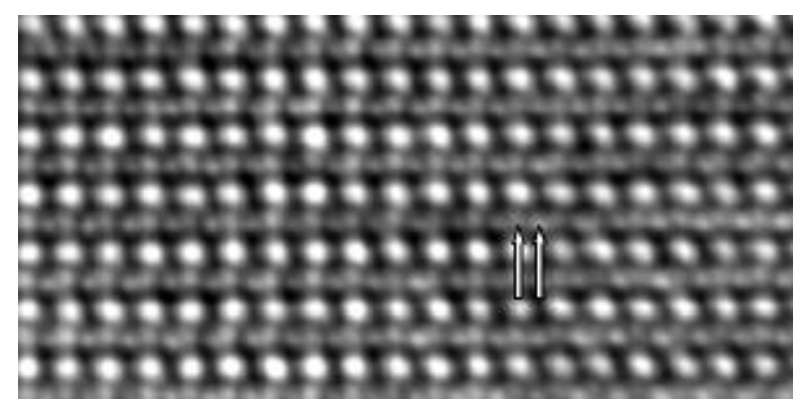

Fig.4 (BaSr)TiO ${ }_{3}[110]$ at $Z=0 / C_{\mathrm{S}}=0$. The separation of $\mathrm{TiO} / \mathrm{O}$ columns (arrows) is $0.14 \mathrm{~nm}$. 